\title{
Post-Stroke Depression: Prevalence, Associated Factors, and Relationship to Disability in a Tertiary Care Center in Sri Lanka
}

\author{
Narmathey Thambirajah ${ }^{1}$ Sunethra Senanayake ${ }^{2}$ Kishara Gooneratne ${ }^{2}$ Chaturi Suraweera ${ }^{3}$ \\ Lakshitha Ranasinghe ${ }^{4(0)}$ Mahesh Kumbukage ${ }^{4(0)}$
}

${ }^{1}$ Ministry of Health, Postgraduate Institute of Medicine, Colombo, Sri Lanka

2 National Hospital Sri Lanka, District General Hospital Hambanthota, Faculty of Medicine, University of Moratuwa, Moratuwa, Sri Lanka

${ }^{3}$ Department of Psychiatry, Faculty of Medicine, National Hospital of

Address for correspondence Mahesh Priyadarahana Kumbukage, MBBS, MSc, MD, Family Health Bureau, 231, De Saram Place, Colombo 10. 01000 , Sri Lanka

Sri Lanka, University of Colombo, Colombo, Sri Lanka

${ }^{4}$ Ministry of Health, Family Health Bureau, Colombo, Sri Lanka

J Neurosci Rural Pract 2022;13:73-79.

\author{
Abstract \\ Keywords \\ - post-stroke \\ depression \\ - risk factors \\ - prevalence \\ - Beck's Depression \\ Inventory \\ - Modified Rankin \\ Score
}

Background and Objectives The prevalence of stroke in urban Sri Lanka is estimated at 10.4 per 1000 and is expected to rise. Post-stroke depression (PSD) is an independent predictor of poor long-term outcomes. It leads to suboptimal rehabilitation, decreased quality of life, and increased mortality and is under-recognized. The main objectives of this study were to estimate the prevalence of depression in stroke, assess factors associated with PSD, and assess the relationship of PSD to disability.

Materials and Methods A descriptive cross-sectional study was conducted at the Neurology and Medical Ward, National Hospital of Sri Lanka. Non-probability, consecutive sampling was used to collect data from patients with ischemic stroke admitted from January 2019 to January 2020. Patients with significant pre-existing depression, cognitive impairment, and language deficits were excluded. A structured, pre-tested interviewer-administered questionnaire was used to assess the prevalence and associated factors of PSD. Beck's Depression Inventory (BDI) was administered 3 months following the stroke to screen for depression. Modified Rankin Score (MRS) was used to assess disability on admission, discharge, and at 3 months.

Results Eighty-one stroke patients were screened. The mean age was 66.6 years ( \pm standard deviation [SD]: 12.5). Male:female ratio was 1.2:1. Depression at 3 months of follow-up was observed in $35.8 \%$ (95\% confidence interval $[\mathrm{CI}]: 25.4-47.2 \%$ ) of participants. Following bivariate analysis, large vessel stroke $(p<0.001)$, cortical stroke $(p<0.001)$, frontal lobe lesions $(p<0.001)$, history of past stroke $(p=0.014)$, and sexual dysfunction ( $p=0.026$ ) were associated with increased risk of PSD. The odds of a person with severe disability developing PSD was 7.9 times more than a person with a published online January 11,2022
DOI https://doi.org/ 10.1055/s-0041-1741504. ISSN $0976-3147$.
(C) 2022. Association for Helping Neurosurgical Sick People. All rights reserved.

This is an open access article published by Thieme under the terms of the Creative Commons Attribution-NonDerivative-NonCommercial-License, permitting copying and reproduction so long as the original work is given appropriate credit. Contents may not be used for commercial purposes, or adapted, remixed, transformed or built upon. (https://creativecommons.org/ licenses/by-nc-nd/4.0/)

Thieme Medical and Scientific Publishers Pvt. Ltd., A-12, 2nd Floor, Sector 2, Noida-201301 UP, India 
less severe disability at discharge from hospital and at 3 months of follow-up (odds ratio $[\mathrm{OR}]=7.9 ; 95 \% \mathrm{Cl}: 2.7-23.3, p=0.000)$.

Conclusions PSD occurs in one-third of strokes, keeping with previous studies. The risk of having PSD is higher among patients with severe disabilities. The difference in risk factors identified compared with previous studies can be attributable to differences in methodology. Identifying risk factors for post-stroke depression is essential to mitigate the poor outcome.

\section{Introduction}

Stroke is a significant contributor to death and disability worldwide and is the third leading cause of death in Sri Lanka. Globally, in 2013, it was estimated that there were 6.5 million deaths from stroke, 25.7 million stroke survivors, and 113 million disabilityadjusted life years (DALY) were lost due to stroke. ${ }^{1}$ In Asia, the overall stroke mortality is higher than that in the Americas, Australasia, and Western Europe. ${ }^{2}$ The prevalence of strokes in urban Sri Lanka was estimated to be 10.4 per 1000 population, ${ }^{3}$ and the number is expected to rise in the future with a rapidly aging population. However, with improvements in treatment and medical care, overall mortality rates are improving with an increasing number of stroke survivors with residual deficits resulting in lost disability-adjusted life years (DALYs). An estimated 1,073.6 DALYs were lost per 100,000 population in Sri Lanka due to stroke compared with 706.6 per 100,000 people in Japan, which has the lowest rate in Asia. ${ }^{1}$

Post-stroke depression (PSD) is an independent predictor of poor long-term post-stroke outcome and has been identified as a condition that leads to unsatisfactory rehabilitation, decreased quality of life, and increased mortality. ${ }^{4-6}$ Thus, it has been described as the "double burden" of stroke. A poststroke disability may have a two-way relationship with PSD, with disability shown as one of the risk factors of PSD. Treatment of PSD has been shown to improve post-stroke patients' activities of daily living. ${ }^{7}$

PSD is an under-recognized sequela of stroke and arriving at a diagnosis can be challenging, especially when cognitive impairment and speech disorders are also present. ${ }^{8}$ There are risks of both under and over-diagnosis. Somatic complaints such as diurnal variation of mood, disturbances in sleep and appetite are used in the depression rating scales and the DSM5 criteria to diagnose depression. ${ }^{9}$ However, they may occur as part of the sequelae of stroke as well. Untreated PSD can become chronic and affect long-term prognosis. ${ }^{10}$

Data on PSD are limited from low-resource settings such as Sri Lanka. This study aimed to determine the prevalence of PSD at 3 months following a stroke, identify risk factors associated with it, and explore the relationship of PSD with the degree of disability.

\section{Materials and Methods}

A descriptive cross-sectional study of patients admitted with ischemic stroke to the Neurology units at the National
Hospital of Sri Lanka (NHSL). This is one of three state-run hospitals that provides healthcare in all medical specialties including neurology services for the Colombo District. Nonprobability, consecutive sampling was used to collect data from patients with ischemic stroke admitted from January 2019 to January 2020. All patients more than 18 years old admitted with ischemic stroke within 2 weeks of the episode confirmed by either computer tomography (CT) scan or by magnetic resonance imaging (MRI) scan were recruited for the study. Patients with transient ischemic attacks, subarachnoid hemorrhage, hemorrhagic stroke, or brain tumors were excluded from the study. Patients who had speech disorders such as dysphasia or dysarthria severe enough to limit effective communication, those who had Mini-Mental State Examination (MMSE) $\leq 19$, previously diagnosed patients with psychiatric illness were excluded from the study.

The eligible participants' information was collected on admission, at discharge, and after 3 months using an interviewer-administered questionnaire by trained medical officers. Data were collected on demographic characteristics, clinical features, risk factors, comorbidities, presence of sexual dysfunction, stroke type, and location. Medical records and investigation results were used to collect additional clinical data. The patient's disability on admission, discharge, and at 3 months was assessed using the Modified Rankin Score (MRS) and National Institutes of Health Stroke Scale (NIHSS). For the final analysis, MRS was considered. Twenty-one-item Beck's Depression Inventory (BDI) scale validated in Sinhala and Tamil was used to evaluate the degree of depression at 3 months follow-up after stroke. ${ }^{11,12}$ The BDI scale ranges from 0 to 43 . Those who scored more than 17 points on this scale were considered depressed. The presence of sexual dysfunction was assessed clinically by the psychiatrist of the team using the DSM 5 criteria defined as the difficulty experienced by the subject or partner/s during any stage of normal sexual activity, including physical pleasure, desire, preference, arousal, or orgasm for both males and females.

The sociodemographic characteristics of the study population were described using frequency distributions and the mean age of the study population. Details of the stroke and risk factors were described using frequency distributions. Variables that did not show normal distributions were presented as medians with interquartile range. As appropriate, statistical testing for associations was performed using 
Pearson's Chi-square test and Fisher's exact test. A probability value of $<0.05$ was considered significant. The severity of the disability at admission, discharge, and at 3 months was compared using a paired $t$-test. Chi-square was used to compare the level of severity and depression. Data analyses were performed using the SPSS version 20.0.

Ethical approval for the study was obtained from the Ethics Review Committee of the Faculty of Medicine, University of Colombo.

\section{Results}

\section{Sociodemographic Characteristics of the Study Population}

Data from a total of 81 patients were analyzed using SPSS version 25.0. The mean age of the study population was 66.6 (standard deviation $[S D]=12.5$ ) ranged from 28 to 88 years with male to female ratio of 44 (54.3\%): 37 (45.7\%). The majority were Sinhalese $(80.2 \%, n=65)$, while $11.1 \%(n=9)$ were Muslims and 8.6\% $(n=7)$ were Tamils. Fifty-six $(69.1 \%)$ were Buddhists, while there were 12 (14.8\%) Catholics, 7 (8.6\%) Islam, and 6 (7.4\%) Hindu. Everybody had received primary education, while $70.4 \%(n=57)$ had completed secondary education and $16 \%(n=13)$ had received tertiary education. One-third of the population $(33.0 \%, n=33)$ monthly income of

Table 1 Sociodemographic distribution of the study population

\begin{tabular}{|l|l|l|l|}
\hline & Variable & No. & $\begin{array}{l}\text { Percentage } \\
(\%)\end{array}$ \\
\hline Gender & Male & 44 & 54.3 \\
\hline & Female & 37 & 45.7 \\
\hline Race $^{\text {a }}$ & Sinhala & 65 & 80.2 \\
\hline & Tamil & 9 & 11.1 \\
\hline & Muslims & 7 & 8.6 \\
\hline Religion $^{\text {b }}$ & Buddhist & 56 & 69.1 \\
\hline & Catholic & 12 & 14.8 \\
\hline & Hindu & 6 & 7.4 \\
\hline & Islam & 7 & 8.6 \\
\hline $\begin{array}{l}\text { Educational } \\
\text { status }\end{array}$ & Primary & 11 & 13.6 \\
\hline & Secondary & 57 & 70.4 \\
\hline & Tertiary & 13 & 16 \\
\hline Employment & Employed & 44 & 54.3 \\
\hline & Unemployed/retired & 37 & 45.7 \\
\hline $\begin{array}{l}\text { Monthly } \\
\text { income }\end{array}$ & $<$ LKR 10,000 & 30 & 37.0 \\
\hline & $<$ LKR 10,001-50,000 & 33 & 40.7 \\
\hline & $>$ LKR 50,001 & 18 & 22.2 \\
\hline
\end{tabular}

${ }^{a}$ There are four main races in Sri Lanka: Sinhala, Tamil, Burgher, and Muslim

${ }^{\mathrm{b}}$ There are four main religions in Sri Lanka: Buddhism, Hinduism, Islam, and Christianity/Catholicism.
10,001-50,000 LKR ( 1 LKR approx. $=0.0050$ USD) while 37\% ( $n=30$ ) were making less than Rs. 10,000 a month ( - Table 1 ).

Thirty-nine (48.1\%) had hypertension alone, whereas 7 (8.6\%) were diabetes alone while 76 (93\%) possessed at least one co-morbidity. More than one-third of the participants were smokers (35.8\%, $n=29$ ). Seventeen patients (21\%) reported sexual dysfunction while two patients $(2.5 \%)$ developed urine incontinence at the follow-up of 3 months after discharge from the hospital.

\section{Stroke Details}

Forty-eight (59.7\%) left hemispheric, 30 (37\%) right hemispheric, one $(1.2 \%)$ bilateral and $2(2.5 \%)$ brainstem lesions were observed among study subjects with 21 (25.7\%) large vessel involvement. Forty-one study subjects (50.6\%) had cerebral atrophy. Amongst all lesions, there were $11(13.6 \%)$ frontal and thalamic 4 (4.9\%), 20 (24.7\%) cortical, 59 (72.8\%) sub-cortical while 2 (2.5\%) had both involvements. Eleven (13.6\%) of the study subjects had a recurrent stroke (-Table 2 ).

\section{Factors Associated with the Development of Post Stroke Depression}

Sociodemographic factors along with details of stroke were compared with the presence of PSD ( - Table 3 ). Bivariate analysis yielded statistically significant associations between PSD and stroke subtype $(p=0.000)$, frontal lesion $(p=0.001)$, past stroke $(p=0.014)$, and sexual dysfunction $(p=0.028)$ following stroke.

Table 2 Stroke details of the study population

\begin{tabular}{|c|c|c|}
\hline & No. & Percentage (\%) \\
\hline \multicolumn{3}{|l|}{ Lateralization } \\
\hline Right hemispheric & 30 & 37.0 \\
\hline Left hemispheric & 48 & 59.3 \\
\hline Bilateral & 1 & 1.2 \\
\hline Brainstem & 2 & 2.5 \\
\hline \multicolumn{3}{|l|}{ Subtype } \\
\hline Large vessel & 21 & 25.9 \\
\hline Small vessel & 60 & 74.1 \\
\hline \multicolumn{3}{|c|}{ Evidence of cerebral atrophy } \\
\hline Yes & 41 & 50.6 \\
\hline No & 40 & 49.4 \\
\hline \multicolumn{3}{|l|}{ Frontal lesion } \\
\hline Present & 11 & 13.6 \\
\hline Absent & 70 & 86.4 \\
\hline \multicolumn{3}{|l|}{ Thalamic lesion } \\
\hline Yes & 4 & 4.9 \\
\hline No & 77 & 95.1 \\
\hline \multicolumn{3}{|l|}{ Cortical lesion } \\
\hline Cortical & 20 & 24.7 \\
\hline Sub-cortical & 59 & 72.8 \\
\hline Both & 2 & 2.5 \\
\hline
\end{tabular}


Table 3 Factors associated with the development of post-stroke depression

\begin{tabular}{|c|c|c|c|c|c|c|}
\hline & \multicolumn{6}{|l|}{ Associated factors/risk factors } \\
\hline \multirow{2}{*}{\multicolumn{2}{|c|}{ Associated factor/Independent variable }} & PSD present & No PSD & \multirow[t]{2}{*}{ OR $(1 / 2)(95 \% \mathrm{Cl})$} & \multirow[t]{2}{*}{$x^{2}$} & \multirow[t]{2}{*}{$p$-Value } \\
\hline & & No. & $\%$ & & & \\
\hline \multirow[t]{2}{*}{ Stage } & $<60$ years & 11 & 11 & \multirow{2}{*}{$\begin{array}{l}0.439 \\
{[0.161-1.197]}\end{array}$} & \multirow[t]{2}{*}{2.649} & \multirow[t]{2}{*}{0.123} \\
\hline & $\geq 60$ years & 18 & 41 & & & \\
\hline \multirow[t]{2}{*}{ Gender } & Male & 19 & 25 & \multirow{2}{*}{$\begin{array}{l}0.487 \\
{[0.19-1.247]}\end{array}$} & \multirow[t]{2}{*}{2.282} & \multirow[t]{2}{*}{0.165} \\
\hline & Female & 10 & 27 & & & \\
\hline \multirow[t]{2}{*}{ Race } & Sinhala & 21 & 44 & \multirow{2}{*}{$\begin{array}{l}2.095 \\
{[0.691-6.353]}\end{array}$} & 1.749 & 0.246 \\
\hline & Others & 8 & 8 & & & \\
\hline \multirow{2}{*}{$\begin{array}{l}\text { Marital } \\
\text { status }\end{array}$} & Married & 23 & 49 & \multirow{2}{*}{$\begin{array}{l}4.261 \\
{[0.978-18.565]}\end{array}$} & \multirow[t]{2}{*}{4.196} & \multirow[t]{2}{*}{0.063} \\
\hline & Others & 6 & $3^{\mathrm{b}}$ & & & \\
\hline \multirow[t]{2}{*}{ Religion } & Buddhists & 18 & 38 & \multirow{2}{*}{$\begin{array}{l}1.659 \\
{[0.630-4.369]}\end{array}$} & \multirow[t]{2}{*}{1.057} & \multirow[t]{2}{*}{0.326} \\
\hline & Others & 11 & 14 & & & \\
\hline \multirow{2}{*}{$\begin{array}{l}\text { Educational } \\
\text { status }\end{array}$} & Up to grade 5 & 5 & 6 & \multirow{2}{*}{$\begin{array}{l}0.626 \\
{[0.173-2.264]}\end{array}$} & \multirow[t]{2}{*}{0.516} & \multirow[t]{2}{*}{0.511} \\
\hline & Secondary and tertiary & 24 & 46 & & & \\
\hline \multirow{2}{*}{$\begin{array}{l}\text { Monthly } \\
\text { income }\end{array}$} & $<\operatorname{LKR} 10,000$ & 24 & 39 & \multirow{2}{*}{$\begin{array}{l}0.625 \\
{[0.198-1.974]}\end{array}$} & \multirow[t]{2}{*}{0.648} & 0.579 \\
\hline & $\geq \operatorname{LKR} 10,000$ & 5 & 13 & & & \\
\hline Subtype & Large vessel & 15 & 6 & 0.122 & 15.65 & 0.001 \\
\hline & Small vessel & 14 & 46 & [0.04-0.373] & & \\
\hline Cerebral atrophy & Yes & 15 & 26 & 0.933 & 0.02 & 1.00 \\
\hline & No & 14 & 26 & [0.376-2.316] & & \\
\hline Frontal lesion & Presence of a frontal lesion & 9 & $2^{b}$ & 0.089 & 11.72 & 0.001 \\
\hline & No frontal lesion & 20 & 50 & {$[0.018-0.448]$} & & \\
\hline Thalamic lesion & Presence of a thalamic lesion & $0^{\mathrm{a}}$ & $4^{\mathrm{b}}$ & $N A^{a}$ & 2.34 & $N / A^{a}$ \\
\hline & No thalamic lesion & 29 & 48 & & & \\
\hline Past stroke & Yes & 8 & $3^{b}$ & 0.161 & 7.55 & 0.014 \\
\hline & No & 21 & 43 & 56] & & \\
\hline Smoking & Yes & 14 & 15 & 2.302 & 3.05 & 0.095 \\
\hline & No & 15 & 37 & [0.896-5.915] & & \\
\hline Sexual dysfunction & Yes & 10 & 7 & 0.296 & 4.96 & 0.028 \\
\hline & No & 19 & 45 & {$[0.98-($} & & \\
\hline
\end{tabular}

${ }^{a}$ Removed from the analysis due to the absence of study subjects who had depression and thalamic lesions.

'Fishers' exact test was applied to arrive at more accurate results for small samples.

\section{Severity of Disability}

The mean disability scores on admission/discharge and discharge/after 3 months were compared using a paired $t$ test. The severity of disability (mean $=1.73$ ) at the time of discharge from the hospital had been significantly less $(p<0.01,95 \%$ confidence interval $[\mathrm{CI}]: 0.28-0.53)$ than that of at the time of admission ( mean $=2.14$ ). The difference in the severity of disability was not significantly associated $\left(\mathrm{X}^{2}=0.089, p=0.081\right)$ with the development of depression at the assessment at 3 months after discharge from the hospital. A person who had no difference in severity had odds of 1.154 of developing depression compared with a person who had a difference in severity at the time of discharge but the result was not statistically significant (95\% CI $=0.45-2.964)$. Similarly, the patient's severity of disability had significantly improved at the end of 3 months of follow-up period. Severity of disability at 3 months (mean $=1.35$ ) had been significantly less ( $p=0.001,95 \% \mathrm{CI}$ : $0.26-$ 0.50 ) than that of at the time of discharge (mean $=1.73$ ).

\section{Comparison of Severity of Disability and Post Stroke Depression}

The severity of disability measured 3 months after discharge from the hospital was dichotomized and compared with depression status. The odds of a person with severe disability developing PSD was 7.912 times more than a person with a less severe disability at the time of 3 months following discharge from the hospital (odds ratio $[\mathrm{OR}]=7.912 ; 95 \%$ CI: 2.683-23.335; $p=0.000$ ) (-Table 4). 
Table 4 Comparison between severity of symptoms and post-stroke depression among the participants

\begin{tabular}{|l|l|l|l|l|l|}
\hline Disability at 3 months after discharge $(\boldsymbol{n}=\mathbf{8 1})$ & \multicolumn{2}{|l|}{ Depression } & OR & $\mathrm{X}^{2}$ & \multirow{2}{*}{$\boldsymbol{p}$-Value } \\
\cline { 2 - 4 } & Yes & No & & \\
\hline Severe disability & $16(19.7 \%)$ & $7(8.6 \%)$ & 7.912 & 15.930 & 0.000 \\
\hline Absence of severe disability & $45(55.5 \%)$ & $13(16.0 \%)$ & {$[2.683-23.335]$} & & \\
\hline
\end{tabular}

\section{Discussion}

The pooled prevalence of PSD of hospital-based stroke patients was reported in a systematic review and was 35\% (95\% CI: $24 \%-46 \%) .{ }^{13}$ This finding was similar to the prevalence of PSD in our study. However, the prevalence rates of PSD can range from $25 \%$ to $79 \%$ in different studies. ${ }^{7,14}$ This is due to the methodological differences between studies. How depression was identified and measured varies in studies; some use PSD-specific scales, whereas others use more general scales. ${ }^{15} \mathrm{~A}$ study that compared screening properties of BDI, Hospital Anxiety and Depression scale-depression subscale (HADS-D), Hamilton Rating Scale for Depression (HAMD), and Montgomery-Asberg Depression Rating Scale (MADRS) in PSD concluded that discriminating abilities of all scales for PSD were good. ${ }^{16}$ However, misclassification was influenced by demographic characteristics and stroke severity, particularly for BDI and HAMD. This might be a limitation in our study as we used BDI for screening. The BDI is a validated screening tool in the Sri Lankan setting, is widely in use, and is a strength for this study. ${ }^{11}$ Inclusion criteria also vary considerably. For example, patients with aphasia or dementia were excluded in certain studies. ${ }^{15}$ As mentioned before the diagnosis of PSD can be difficult especially in patients with aphasia and other cognitive deficits such as memory loss, agnosia, and apraxia. This is due to the symptoms of stroke or depression overlapping, making them indistinguishable from each other. Moreover, it is essential to differentiate PSD from post-stroke apathy, which affects $\sim 40 \%$ of stroke patients and is characterized by reduced motivation/goal-directed behavior, and impaired emotion and cognition. ${ }^{17}$ It is for this reason that patients with such deficits were excluded from our study. Inclusion criteria also differed with regard to the target populations, age groups, or phases of the disease (acute: within 3 months versus chronic: after 3 months). ${ }^{15}$ The setting in which stroke patients are assessed can also have an impact on the prevalence of PSD. For instance, the rate of PSD in the community can be lesser than that in a hospital-based setting. ${ }^{15,18}$

The frequency of PSD is the highest in the first year after the stroke and declines after 12 months. ${ }^{17}$ A large Danish study with $>150,000$ patients hospitalized for stroke observed that the risk was particularly high in the first 3 months after the vascular event $(\mathrm{OR}=8.99 ; 95 \% \mathrm{CI}:=8.61-9.39){ }^{19}$ This may be due to the following factors: higher disability, greater medical burden, and lower independence due to stroke-related deficits. Furthermore, social support is supposed to have a protective effect reducing PSD chance through emotional support, motivation for treatment, and support with daily functioning. PSD in our cohort was associated with a higher degree of physical disability at 3 months, thus causing a lower degree of independence in such patients with stroke. This reinforces the findings of previous studies and lends support to the above premise, which explains the higher prevalence of PSD in our cohort. ${ }^{20}$

Stroke laterality has been proposed as a risk factor for PSD. However, rates of PSD based on laterality remain inconsistent. Mitchell et al demonstrated a statistically significant difference, with PSD occurring in 34\% of left-sided lesions versus $18 \%$ of right-sided lesions ( $\mathrm{OR}=1.50$; $95 \% \mathrm{CI}$ : 1.29 1.74). ${ }^{21}$ However, although Douven et al found that those with left-sided stroke had a $26 \%$ higher risk of developing PSD, this difference was not statistically significant (OR $=1.26$; 95\% CI: $0.95-1.67) .{ }^{22}$ We did not find a statistical difference between PSD occurring in dominant and nondominant hemisphere strokes. However, this may be due to strokes with dysphasia and severe cognitive deficits (often observed in left or dominant hemisphere strokes) being excluded from the study.

Large and multiple strokes are predictive of higher frequencies of PSD, suggesting that greater neurological tissue loss serves as a risk factor for PSD. ${ }^{23}$ Our study observed this with large artery stroke and previous history of stroke having a positive association with PSD. This was observed in previous studies as well, though some studies have contradicted this finding. ${ }^{23}$ More recent studies have implicated subcortical white matter involvement as a risk factor for PSD, which was not observed in our study. ${ }^{23}$

Previous studies have suggested that frontal lobe/anterior strokes were associated with higher rates of PSD when compared with strokes in other areas. ${ }^{24} \mathrm{~A}$ recent metaanalysis found that this relationship was statistically significant in the post-acute phase, defined as any time from 15 days to 6 months $(\mathrm{OR}=1.72$ and $95 \% \mathrm{CI}=1.34-2.41$ for the frontal lobe). ${ }^{22}$ Furthermore, several DTI studies in patients with a depressive disorder report structural alterations of fronto-striato-thalamic pathways and anterior interhemispheric connections. These are pathways in connecting brain regions and networks involved in tasks such as decision-making, emotional regulation, and reward processing, which may explain the higher association of PSD with lesions involving the frontal region. ${ }^{25}$ Such lesions can lead to interruption of monoaminergic pathways implicated in mood regulation. ${ }^{26}$ Our study observed a positive association with frontal lobe lesions with PSD similar to previous studies although we did not observe a positive association with thalamic lesions. The difference observed could be due to limitations in sample size (only four thalamic strokes were 
observed), inaccuracies in visual assessment of the lesion (more accurate techniques of lesion analysis include: lesion segmentation, methods of brain registration to standard space, voxel-based statistics) ${ }^{23}$ and certain strokes being excluded due to exclusion criteria.

Several studies found a strong correlation between PSD, anxiety, and sexual dysfunction (SD), as seen in our study. ${ }^{27}$ Post-stroke SD is quite common with a prevalence ranging from 20 to $75 \%$ and it is commoner in males. The etiology of SD can be multifactorial following stroke. Risk factors for post-stroke SD include lesions involving the thalamus, nondominant parietal lobe, insular and cerebellum, physical dysfunction, PSD itself, co-morbidities such as diabetes, ischemic heart disease, and hypertension affecting the vascular endothelium supplying the sexual organs, and autonomic dysfunction. ${ }^{27}$

We did not observe significant associations with age, sex, and level of education with PSD. Evidence of age as a risk factor for PSD is conflicting. Two previous studies reported that age $<70$ was a risk factor for PSD ${ }^{28,29}$. However, two other studies found that older individuals were at higher risk for PSD. ${ }^{19,30}$ Studies have found that female sex and lower educational level were risk factors for developing PSD ${ }^{17,31}$.

Personal history of psychiatric illness, family history of psychiatric disorders, and a higher degree of neurosis are pre-existing conditions that predict PSD. ${ }^{17}$ However, such patients in our study were excluded as it was difficult to differentiate depression following a stroke from pre-existing depression. A caveat with this means that individuals with resolved pre-existing depression who experienced PSD would have been excluded, leading to underestimating the true prevalence of PSD.

Limitations to our study include the restricted sample size, attributable to attrition at 3-month follow-up, as the screen for depression was conducted during this time. Furthermore, the exclusion of subjects with severe cognitive impairment and language deficits due to the ability to comprehend the screening questions and the difficulty in differentiating actual depressive symptoms from symptoms due to the stroke itself, such as apathy, also contributed to a smaller sample size. Thus, the true prevalence of PSD may have been an underestimation. Furthermore, stroke with severe disability was excluded from the study; thus, correlating the degree of disability with PSD occurrence may not be accurate. While stating limitations in sample size in our study, recent studies highlighting the prevalence of PSD in cohorts of patients in Saudi Arabia and China published data on 50 and 91 stroke subjects respectively. ${ }^{32,33}$ The lack of prevalence studies in the South Asian region also adds further value to the data in the present study despite its limitations.

An earlier study done in Sri Lanka in a similar setting revealed a prevalence of PSD $28 \%$ similar to our study. ${ }^{34}$ They found that female gender, duration of pre-existent hypertension, Barthel index (degree of disability), and temporal lobe involvement were independently associated with PSD. The main methodological differences in this study were: depression was assessed within one month of stroke, de- pression was diagnosed by a single psychiatrist based on the ICD-10 criteria, and patients with both ischemic and hemorrhagic strokes were included. Such differences probably explain the differences in risk factors.

\section{Conclusion}

PSD is often underrecognized and is a barrier to optimal rehabilitation in clinical practice. This study highlights the prevalence of PSD in a cohort of stroke patients in the hospital setting and mirrors the already observed prevalence in previous studies conducted in similar settings. This study's risk factors for PSD include the severity of the disability, large artery stroke, cortical stroke, frontal lobe lesions, past history of stroke, and sexual dysfunction. Differences in risk factors for PSD in different studies emphasize the need for large-scale multi-centered studies with a consensus on the diagnosis of PSD and studies of patients in different settings (hospital, community, etc.). This would allow for more accurate identification of risk factors for PSD, which is vital to mitigate poor stroke outcomes.

\section{Ethical Approval \\ Ethical approval for the research was obtained from the Ethics Review Committee at the Medical Faculty, Univer- sity of Colombo.}

\section{Funding \\ None}

Conflict of Interest

None declared.

\section{Acknowledgments}

The authors thank the medical officers who were instrumental in obtaining the data for the study.

\section{References}

1 Feigin VL, Krishnamurthi RV, Parmar P, et al; GBD 2013 Writing Group GBD 2013 Stroke Panel Experts Group. Update on the Global Burden of Ischemic and Hemorrhagic Stroke in 1990-2013: the GBD 2013 Study. Neuroepidemiology 2015;45(03):161-176

2 Venketasubramanian N, Yoon BW, Pandian J, Navarro JC. Stroke epidemiology in South, East, and South-East Asia: a review. J Stroke 2017;19(03):286-294

3 Chang T, Gajasinghe S, Arambepola C. Prevalence of stroke and its risk factors in Urban Sri Lanka: population-based study. Stroke 2015;46(10):2965-2968

4 Haghgoo HA, Pazuki ES, Hosseini AS, Rassafiani M. Depression, activities of daily living and quality of life in patients with stroke. J Neurol Sci 2013;328(1-2):87-91

5 Tsuchiya K, Fujita T, Sato D, et al. Post-stroke depression inhibits improvement in activities of daily living in patients in a convalescent rehabilitation ward. J Phys Ther Sci 2016;28(08): 2253-2259

6 Hackett ML, Yapa C, Parag V, Anderson CS. Frequency of depression after stroke: a systematic review of observational studies. Stroke 2005;36(06):1330-1340

7 Robinson RG, Spalletta G. Poststroke depression: a review. Can J Psychiatry 2010;55(06):341-349 
8 Fedoroff JP, Starkstein SE, Parikh RM, Price TR, Robinson RG. Are depressive symptoms nonspecific in patients with acute stroke? Am J Psychiatry 1991;148(09):1172-1176

9 Tolentino JC, Schmidt SL. DSM-5 criteria and depression severity: implications for clinical practice. Front Psychiatry 2018;9:450

10 Lai YJ, McCullough LD. Poststroke depression: pathophysiology and treatment strategies. In: Neurobiology of Depression. Elsevier; 2019:197-205

11 Rodrigo A, Kuruppuarachchi KALA, Pathmeswaran A. Validation of the Beck Depression Inventory II among the Sinhalese speaking population in Sri Lanka. Sri Lanka J Psychiatry 2015;6(02):20

12 Beck A, Steer R, Brown G. Beck Depression Inventory II (BDI-II). https://pearsonclinical.in/solutions/beck-depression-inventoryii-bdi-ii/

13 Hackett ML, Pickles K, Part I. Part I: frequency of depression after stroke: an updated systematic review and meta-analysis of observational studies. Int J Stroke 2014;9(08):1017-1025

14 Lökk J, Delbari A. Management of depression in elderly stroke patients. Neuropsychiatr Dis Treat 2010;6:539-549

15 Espárrago Llorca G, Castilla-Guerra L, Fernández Moreno MC, Ruiz Doblado S, Jiménez Hernández MD. Post-stroke depression: an update. Neurologia 2015;30(01):23-31

16 Kang H-J, Stewart R, Kim J-M, et al. Comparative validity of depression assessment scales for screening poststroke depression. J Affect Disord 2013;147(1-3):186-191

17 Medeiros GC, Roy D, Kontos N, Beach SR. Post-stroke depression: A 2020 updated review. Gen Hosp Psychiatry 2020;66:70-80

18 Chau JP-C, Thompson DR, Chang AM, et al. Depression among Chinese stroke survivors six months after discharge from a rehabilitation hospital. J Clin Nurs 2010;19(21-22):3042-3050

19 Jørgensen TSH, Wium-Andersen IK, Wium-Andersen MK, et al. Incidence of depression after stroke, and associated risk factors and mortality outcomes, in a large cohort of Danish patients. JAMA Psychiatry 2016;73(10):1032-1040

20 Kutlubaev MA, Hackett ML, Part II. Part II: predictors of depression after stroke and impact of depression on stroke outcome: an updated systematic review of observational studies. Int J Stroke 2014;9(08):1026-1036

21 Mitchell AJ, Sheth B, Gill J, et al. Prevalence and predictors of poststroke mood disorders: a meta-analysis and meta-regression of depression, anxiety and adjustment disorder. Gen Hosp Psychiatry 2017;47:48-60
22 Douven E, Köhler S, Rodriguez MMF, Staals J, Verhey FRJ, Aalten P. Imaging markers of post-stroke depression and apathy: a systematic review and meta-analysis. Neuropsychol Rev 2017;27(03): 202-219

23 Nickel A, Thomalla G. Post-stroke depression: impact of lesion location and methodological limitations-a topical review. Front Neurol 2017;8:498

24 Robinson RG, Jorge RE. Post-stroke depression: a review. Am J Psychiatry 2016;173(03):221-231

25 Chen G, Hu X, Li L, et al. Disorganization of white matter architecture in major depressive disorder: a meta-analysis of diffusion tensor imaging with tract-based spatial statistics. Sci Rep 2016;6(01):21825

26 Shao X, Zhu G. Associations among monoamine neurotransmitter pathways, personality traits, and major depressive disorder. Front Psychiatry 2020;11:381

27 Park J-H, Ovbiagele B, Feng W. Stroke and sexual dysfunction - a narrative review. J Neurol Sci 2015;350(1-2):7-13

28 Carota A, Berney A, Aybek S, et al. A prospective study of predictors of poststroke depression. Neurology 2005;64(03):428-433

29 Li J, Zhao YD, Zeng JW, Chen XY, Wang RD, Cheng SY. Serum brainderived neurotrophic factor levels in post-stroke depression. J Affect Disord 2014;168:373-379

30 Cheng LS, Tu WJ, Shen Y, Zhang LJ, Ji K. Combination of highsensitivity C-reactive protein and homocysteine predicts the post-stroke depression in patients with ischemic stroke. Mol Neurobiol 2018;55(04):2952-2958

31 Backhouse EV, McHutchison CA, Cvoro V, Shenkin SD, Wardlaw JM. Cognitive ability, education and socioeconomic status in childhood and risk of post-stroke depression in later life: a systematic review and meta-analysis. PLoS One 2018;13(07): e0200525

32 Ahmed ZM, Khalil MF, Kohail AM, Eldesouky IF, Elkady A, Shuaib A. The prevalence and predictors of post-stroke depression and anxiety during COVID-19 pandemic. J Stroke Cerebrovasc Dis 2020;29(12):105315

33 Zhang WN, Pan YH, Wang XY, Zhao Y. A prospective study of the incidence and correlated factors of post-stroke depression in China. PLoS One 2013;8(11):e78981

34 Isuru A, Hapangama A, Ediriweera D, Samarasinghe L, Fonseka M, Ranawaka U. Prevalence and predictors of new onset depression in the acute phase of stroke. Asian J Psychiatr 2021;59:102636 